\title{
CLINICAL EVALUATION OF USING CORTICAL LAMINA XENOGRAFT IN RECONSTRUCTION OF ORBITAL FLOOR
}

\author{
Haitham H. Sakr ${ }^{1 *} M D S$, Ahmed M. Shaaban² $P h D$, Tarek M. Aly² $P h D$, Samraa Elshiekh² $P h D$.
}

\begin{abstract}
INTRODUCTION: The orbital floor is one of the most frequently injured areas during facial trauma. Their proper treatment is crucial for prevention of orbital complications as diplopia, limitation of ocular motility and enophthalmos. Cortical lamina is a collagenated cortical bone of heterologous origin that is used in grafting of non-load-bearing areas.

OBJECTIVE: To evaluate clinically the use of cortical lamina in the reconstruction of orbital floor defects.

Materials and Methods: Ten patients with orbital floor defects less than $2 \mathrm{~cm}$ were associated with one or more of the following complications; binocular diplopia, enophthalmos, limitation of ocular motility or infra orbital nerve dysfunction, were indicated for orbital reconstruction using cortical lamina xenograft. The follow-up schedule was 1 week and 1, 3, 6 months postoperatively.

RESULTS: Orbital complications had been resolved in all patients. Only infra orbital nerve dysfunction continued in 7 patients where 3 of patients recovered after 3 months and the other 4 have recovered after 6 months.

CONCLUSIONS: Cortical lamina xenograft is a good alternative for the reconstruction of orbital floor defects less than $2 \mathrm{~cm}$ due to its plasticity and biocompatibility without any donor site morbidity.

KEYWORDS: Orbital floor, Lamina, Xenograft, Orbital complications

RUNNING TITLE: Cortical lamina in reconstruction of orbital floor defects.
\end{abstract}

1 Assistant lecturer of Oral and maxillofacial surgery, Faculty of Dentistry, Pharos University, Alexandria, Egypt.

2 Professor of Oral and maxillofacial surgery, Faculty of Dentistry, Alexandria University, Alexandria, Egypt.

*Corresponding author

E-mail: haitham.sakr@yahoo.com

\section{INTRODUCTION}

The orbital floor is one of the most frequently injured areas following facial trauma (1).

Orbital floor fractures might lead to several problems including; unfavorable aesthetics, diplopia, gaze restriction, enophthalmos, and infraorbital nerve dysfunction $(2,3)$.

The goals of orbital floor reconstruction are to free the prolapsed orbital fat and entrapped ocular muscle in addition, to span the defect (4). Implant materials that can be used to restore orbital floor defects include autograft, alloplast, allograft and Xenografts $(5,6)$.

Autogenous grafts such as cranial cortical bone, iliac bone, olecranon, conchal auricular cartilage, dura mater, and fascia lata, have the advantages of biocompatibility and lower potential for infection, exposure, and foreign body reaction (7). On the other hand, morbidity of the donor site, problems with providing desired plasticity and prolonged surgical time, are the main disadvantages of using autografts (8).

The allogenic materials that have been used for this purpose are human dura mater, fascia lata and lyophilized cartilage. Reports on the risk of infectious transmitted diseases (such as Creutzfeldt-Jakob disease) have been associated with cadaveric allograft (9).

Alloplastic materials such as polydiaxanone, polyethylene, methyl methacrylate, and titanium implants are cost-effective, readily available, and easily conformed to the contour of the orbital floor. Potential drawbacks for the use of alloplastic material are relatively low, but they include; extrusion, globe elevation, foreign body reaction infection and late proptosis secondary to capsular hemorrhage (10).

Xenogeneic biomaterials display a similar morphology as human bone and have the potential of being resorbed. They have the potential to be a viable substitute to autograft and allograft (11-15). Xenograft materials of porcine origin have provoked a great deal of research to assess their potential as a substitute for osseous grafts. It provides biocompatible, bioabsorbable, and osteoconductive matrix $(16,17)$.

\section{MATERIALS AND METHODS}

The prospective clinical trial has been applied between 2017 and 2018 on ten patients. Patients were admitted to the Oral and Maxillofacial Surgery Department, Faculty of Dentistry, Alexandria University; suffered from orbital floor fractures indicated for surgical repair.

The research protocol was approved by the Ethical Committee of the Faculty of dentistry Alexandria University, and an informed consent was obtained from each patient after providing detailed information and description of the study. Patients suffering from orbital floor fractures; either bilaterally or unilaterally or associated with other maxillofacial injuries were included in the study. Patients were diagnosed clinically with ophthalmology consultation and radiographically using computed tomography (CT). (Figure 1) 
The clinical indications for surgery included; binocular diplopia, enophthalmos more than $2 \mathrm{~mm}$ measured by Hertel exophthalmometry (it measures the distance between the lateral orbital rim to corneal apex), restriction of ocular motility and infraorbital nerve dysfunction. The radiographic indications for surgery included defect of orbital floor less than $2 \mathrm{~cm}$, herniation of orbital fats in the maxillary sinus or entrapment of extraocular muscles.
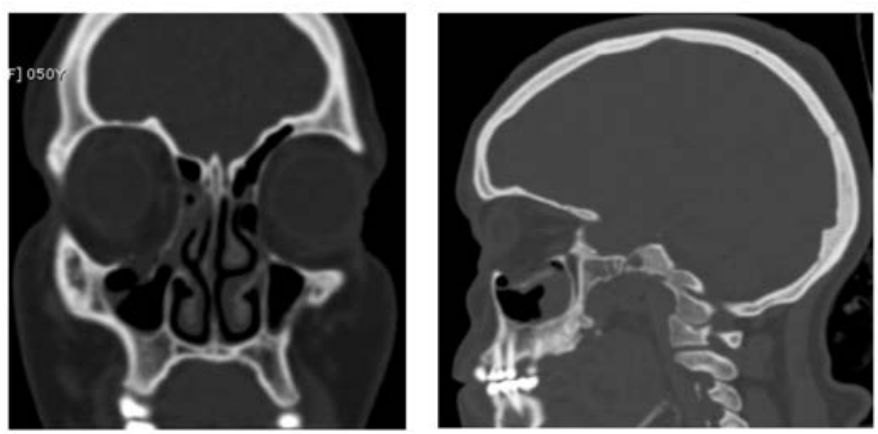

Figure (1): Sagittal and coronal views of orbital floor defect.

We used porcine cortical lamina for orbital floor reconstruction. Porcine Cortical lamina (Tecnoss Medical Devices, Giaveno, Italy) (Figure 2) with dimensions of $35 \mathrm{x}$ $35 \times 1 \mathrm{~mm}$, made of cortical bone of heterologous origin, which undergoes a process of superficial decalcification, maintaining the typical consistency of the bone tissue from which it originates.

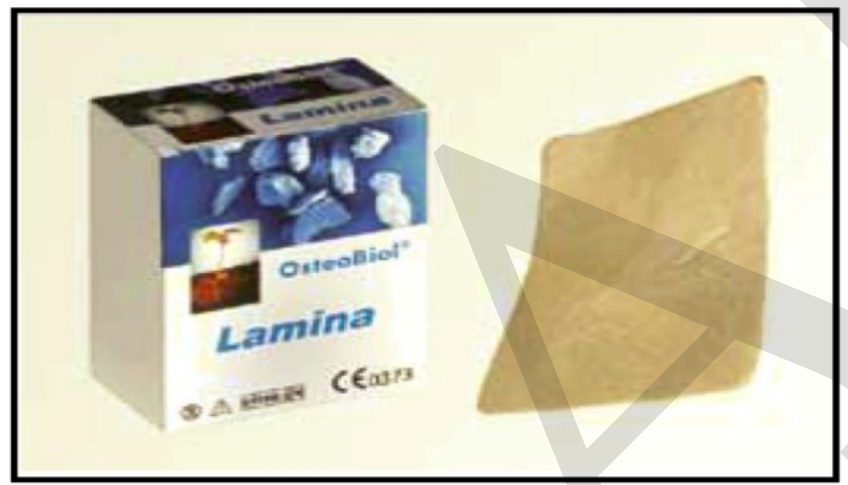

Figure (2): Cortical lamina.

Orbital complications were detected preoperatively and revised 1 week, 1month, 3 months and 6 months postoperatively. Enophthalmos was measured using Hertel exophthalmometry, diplopia and infraorbital nerve dysfunction were detected subjectively by asking the patient about double vision of an object in the former complication and numbness in the areas supplied by terminal branches of infraorbital nerve in the latter complication. Examination of eye movement in different gazes to detect any restriction in ocular muscle movement.

Surgery was carried out under general anesthesia. Before surgical intervention forced duction test was done to examine the limitation of ocular motility. The approach to orbital

floor was through an infraorbital or subtarsal incision. Infraorbital rim and the orbital floor were exposed by subperiosteal dissection. Entrapped soft tissue was repositioned into the orbit and small bony fragments were removed. Suture pack was used as a template to determine the shape and size of the defect. Once the size and shape of the defect were determined, the preparation of dehydrated cortical lamina was initiated. (Figure 3)

The lamina was hydrated in sterile physiological solution at room temperature to be easily handled, as recommended by the manufacturer, and was later shaped according to the template. Care was taken to make the lamina just larger than the size of the defect to achieve reliable placement of the graft over the defect. The lamina was inserted over the defect (Figure 4) and forced duction test was reapplied to ensure freeing of all herniated soft tissue. After securing the graft in position, the wound was closed in two layers (muscle and skin). Temporary suspension suture was applied in the lower eyelid to prevent ectropion. Postoperative CT radiograph was taken. (Figure 5)
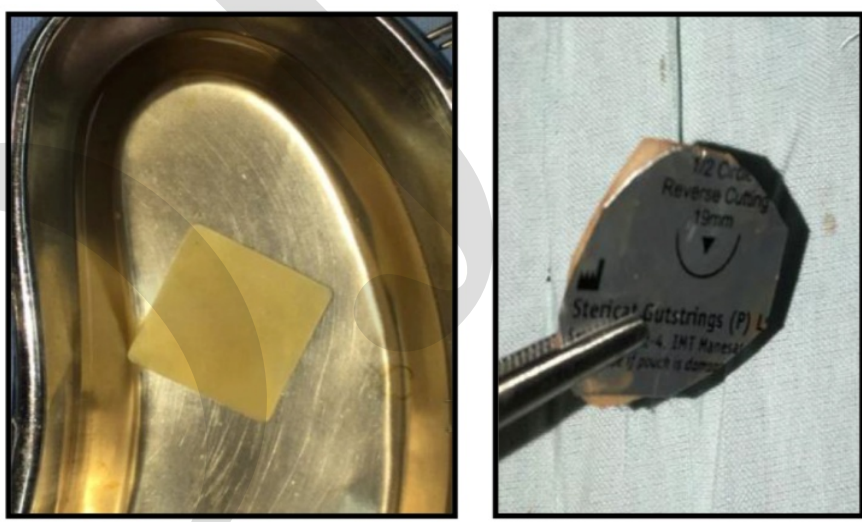

Figure (3): Hydration of cortical lamina and using suture pack as a template to the size orbital defect.
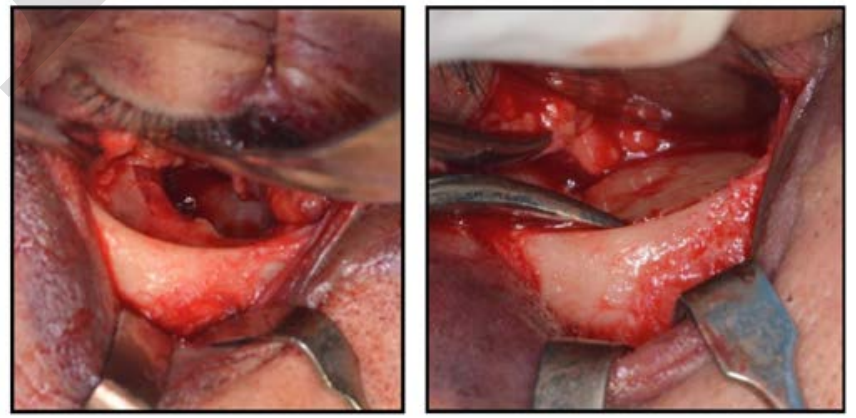

Figure (4): The orbital defect and lamina in place covering the defect. 

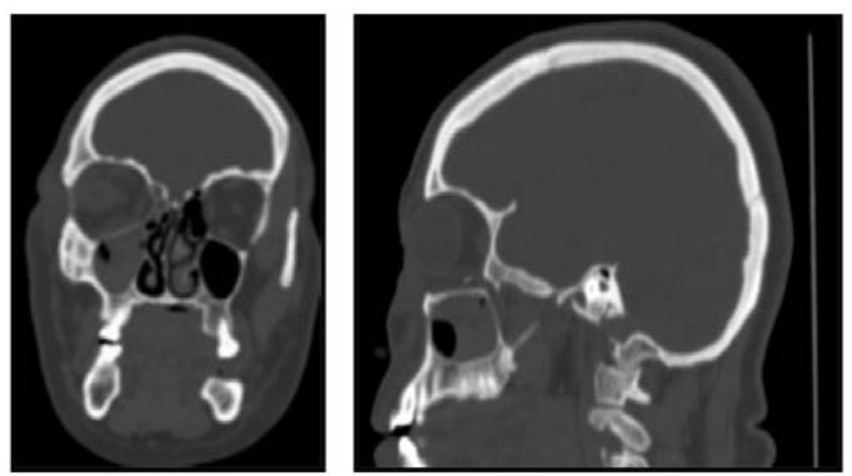

Figure (5): Proper alignment of the lamina appeared in the Postoperative coronal and sagittal CT views.

\section{RESULTS}

Cortical lamina was used for orbital floor reconstruction in 10 patients (9 men and 1 woman; mean age 34 years; range 16-50 over a period of 1 year between 2017 and 2018.

The causative factors for trauma were road traffic accident in six cases, personal violence in two cases, fall in one case and work-related trauma in one case. Orbital floor defect associated with zygomatic complex fracture in five patients, blow out fracture in three patients, zygomatic complex with mandibular symphysial and condylar fracture in one patient and frontal bone with zygomatic complex fracture in one patient. Early intervention within 2 weeks was done for all patients.

Preoperatively, physical examination findings; infraorbital nerve paresthesia in 7 patients, enophthalmos in four patients, gaze restriction in upward direction in 3 patients and binocular diplopia in two patients. None of the patients showed impaired visual acuity preoperatively or postoperatively. Postoperatively binocular diplopia, gaze restriction and enophthalmos were improved in all the patients throughout the follow up period. Seven patients had preoperative infraorbital nerve paresthesia that resolved in 3 patients after 3 months and resolved in the other 4 after 6 months (Figure 6) (Table1). There has been no graft extrusion, resorption, or displacement during the follow-up period.

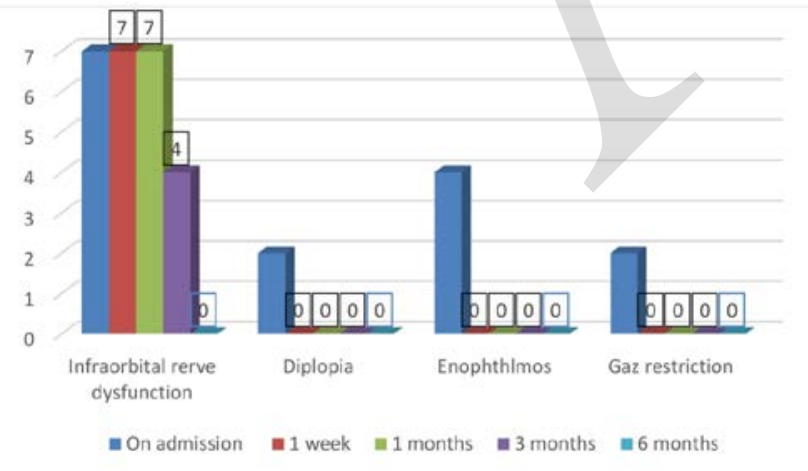

Figure (6): Postoperative recovery of patients from orbital complications.

\section{DISCUSSION}

Orbital floor reconstruction has been and still a matter of controversy in literature with respect to the indication, timing, surgical technique, access and reconstruction materials used $(18,8)$. Orbital floor fractures may lead to enophthalmos, limitation of eye movements, and binocular diplopia due to periocular muscle entrapments that indicate surgical interference and orbital floor repair $(19,20)$.

The main goals of the treatment are to reposition the entrapped orbital content, restore the shape and the volume of the orbit to achieve acceptable functional and cosmetic results and prevent late complications (7).

The best material used in orbital floor reconstruction has been a controversial topic. Autologous, alloplastic and allogeneic materials have been used each with its own advantages and disadvantages (6).

Xenogeneic materials, mainly of equine and porcine origin, are widely used as bone substitutes in reconstructive surgery

for orthopedic surgery, ophthalmology, dentistry as well as maxillofacial surgery (21).

Tecnoss cortical Lamina is a collagenated bone material. It is made of cortical bone of heterologous origin, which undergoes a process of superficial decalcification, maintaining the typical consistency of the bone tissue from which it originates. It is preshaped sheets that are reported to be manufactured using biotechnology that prevents the ceramization phase of natural bone and also preserves tissue collagen, thus allowing an osteoclastic type of remodeling of the biomaterial which mimics physiological bone turnover (22). After the process of superficial decalcification, it acquires an elastic consistency. Our four patients have suffered from enophthalmos show complete resolution 1 week post operatively due to proper fracture reduction and proper orbital floor gentle curvature restoration using the advantage of slight malleability of hydrated cortical lamina (23).

Although Titanium Mesh Implants (TMI) are commonly used for orbital floor fracture reconstruction but cortical lamina overcomes a lot of titanium mesh disadvantages. It can be easily shaped, contoured and trimmed without any sharp edges that overcome problem of the sharp edges of titanium mesh if not probably trimmed. Furthermore, the relative stiffness of titanium mesh may become a potential disadvantage in case of recurrent injuries as the implant may become distorted and behave as a penetrating foreign body able to threaten the intraorbital content (eyeball, nerves, vessels) (24).

Titanium mesh is a non-absorbable material it may cause late adverse effects including infection, implant corrosion and toxicity due to metal ion release (25).

Concerning biocompatibility cortical lamina proved to be good with no adverse or foreign-body reaction. This finding coincides with finding of Rinna et al. (26), who used different grafting materials including autogenous graft, allograft, xenograft and alloplastic material on 379 patients have suffered from blowout fracture and Nappe et al. (27), who used allograft, xenograft and alloplastic graft as bone substitute materials in sinus lifting procedure. 
One of the main requirements of material used in orbital floor reconstruction is being radio-opaque as radio-opacity enable the clinician to locate the graft in the CT radiograph which is one of the main advantages of the lamina $(4,28)$.

In our study all patients with infraorbital nerve dysfunction were completely recovered 6 months post operatively. Haapanen et al. (29), demonstrated complete recovery of infraorbital nerve dysfunction in $44 \%$ of patients 6 months post-operatively, while Wang et al. (30), demonstrated complete recovery in $89 \%$ of patients 6 months postoperatively; these wide variations in infraorbital nerve dysfunction recovery percentages of different studies may be related to the type of primary trauma to the nerve (pinching, laceration, or traction) which has a key role in the nerve recovery.

\section{CONCLUSIONS}

The findings of this study provide evidence that porcine cortical lamina is a good and safe alternative for the reconstruction of orbital defects less than $2 \mathrm{~cm}$, due to their plasticity and biocompatible structure without donor site morbidity.

\section{Conflict of interest}

The authors declare that they have no conflict of interest.

\section{REFERENCES}

1. Yavuzer R, Tuncer S, Başterzi Y, Işik I, Sari A, Latifoğlu O. Reconstruction of orbital floor fracture using solventpreserved bone graft. Plastic and Reconstructive Surgery 2004; 113(1):34-44.

2. Sakakibara S, Hashikawa K, Terashi H, Tahara S. Reconstruction of the orbital floor with sheets of autogenous iliac cancellous bone. Journal of Oral and Maxillofacial Surgery: Official Journal of the American Association of Oral and Maxillofacial Surgeons 2009; 67(5):957-61.

3. Piombino $\mathrm{P}$, Spinzia $\mathrm{A}$, Abbate $\mathrm{V}$, Bonavolontà $\mathrm{P}$, Orabona GD, Califano L. Reconstruction of small orbital floor fractures with resorbable collagen membranes. The Journal of Craniofacial Surgery 2013; 24(2):571-4.

4. Baino F. Biomaterials and implants for orbital floor repair. Acta Biomaterialia 2011; 7(9):3248-66.

5. Tabrizi R, Ozkan TB, Mohammadinejad C, Minaee N. Orbital floor reconstruction. The Journal of Craniofacial Surgery 2010; 21(4):1142-6.

6. Yeşiloğlu N, Sirinoğlu H, Sarici M, Temiz G, Filinte GT. A New Option for the Reconstruction of Orbital Floor Defects: The Olecranon Bone Graft. Annals of Plastic Surgery 2015; 75(4):401-6.

7. Nowinski D, Messo E, Hedlund A. Treatment of orbital fractures: evaluation of surgical techniques and materials for reconstruction. The Journal of Craniofacial Surgery 2010; 21(4):1033-7.

8. Mok D, Lessard L, Cordoba C, Harris PG, Nikolis A, Mok $\mathrm{D}$, et al. A review of materials currently used in orbital floor reconstruction. Canadian Journal of Plastic Surgery 2004; 12(3):134-40.

9. Prichard V, Thadani R, Kalb E. Rapidly progressive dementia in a patient who received a cadaveric dura mater graft. JAMA 1987;257:1036.

10. Bratton M, Durairaj D. Orbital implants for fracture repair. Curr Opin Ophthalmol 2011;22(5):400-6.

11. Webster k. Orbital floor repair with lyophilized porcine dermis. Oral Surg Oral Med Oral pathology.1988;65:161-4.

12. Schlickerwie W, Paul C. Experimentelle Untersuchungen Zum Knochersatz mit mit bovinem Apatit. Hefte zu Unfallsheilkunde 1991; 216:59-69.

13. Fukuta K, Har-Shai Y, Collares V, Lichten B, Jackson T. Comparison of inorganic bovine mineral particles with porous hydroxyapatite granulaes and cranial bone dust in the reconstruction of full thickness skull defects. J Craniofac Surg 1992; 3:25-29.

14. Klinge B, Alberius $\mathrm{P}$, Isaksson S, Jönsson J. Osseous response to implanted natural bone mineral and synthetic hydroxyapatite ceramics in the repair of experimental skull bone defects. J Oral Maxillofac Surg 1992; 80:241- 249.

15. Chowdhury S, Saprubrig B,Awasthi P. Clinico-radiographic evaluation of xenografts in maxillofacial surgery medical journal armed forces india volume 57 , issue 4, october 2001, pages 281-284.

16. Borie C, Cramer V, Phan-Thanh L, Vaillant C, Bequet L, Makowka L, et al. Microbiological hazards related to xenotransplantation of porcine organs into man. Infect Control HospEpidemiol. 1998;19(5):355-65.

17. Costa F, Robiony M, Politi M. Stability of Le Fort I osteotomy in maxillary advancement: Review of the literature. Int J Adult Orthod Orthognath Surg1999;14(3):207-13.

18. Bhatnagar S, Khare P. Reconstruction of orbital floor fracture using porous polyethylene mesh.Int $\mathrm{J}$ Med Res Rev 2014; 2: 1.

19. Mok D, Lessard L, Cordoba C, Harris G, Nikolis A. A review of materials currently used in orbital floor reconstruction.Can J Plast Surg. 2004; 12:134.

20. Burnstine A. Clinical recommendations for repair of isolated orbital floor fractures. Ophthalmology 2002; 109:1207-10

21. Nowinski D, Messo E, Hedlund A. Treatment of orbital fractures: evaluation of surgical techniques and materials for reconstruction. J Craniofac Surg. 2010;21:1033Y1037.

22. Kolmas J, Szwaja M, Kolodziejski W: Solid-state NMR and IR characterization of commercial xenogeneic biomaterials used as bone substitutes. J Pharm Biomed Anal 61: 136e141, 2012.

23. Timothy A, Golden A. Orbital Anatomy for the Surgeon. Oral Maxillofac Surg Clin North Am. 2012 Nov; 24(4): 525-36.

24. Foletti M, Martinez V, Haen P, Godio-Raboutet Y, Guyot L, Thollon L. Finite element analysis of the human orbit. Behavior of titanium mesh for orbital floor reconstruction in case of trauma recurrence. J Stomatol Oral Maxillofac Surg. 2019 Apr;120(2):91-94.

25. Andreiotelli M, Wenz J, Kohal J: Are ceramic implants a viable alternative to titanium implants? A systematic literature review. Clin Oral Implants Res 20(Suppl. 4): 32e47, 2009.

26. Rinna C, Ungari , Saltarel A, Cassoni A, Reale G. Orbital Floor Restoration. Journal of Craniofacial Surgery. 
16(6):968-972, November 2005.

27. Nappe E, Rezu C, Montecinos A, Donoso A, Vergara J, Martinez B. Histological comparison of an allograft, a xenograft and alloplastic graft as bone substitute materials. J Osseointegr 2016;8(2):20-26.

28. Dubois L, Steenen A, Gooris J, Mourits P, Becking G. Controversies in orbital reconstruction-II. Timing of orbital reconstruction in trauma: a systematic review. Int J Oral Maxillofac Surg 2015;44:433-40.

29. Haapanen A, Thorén H, Apajalahti S , SuominenA, Snäll J. Neurosensory recovery after trauma to the orbital floor: a prospective trial with dexamethasone. British Journal of Oral and Maxillofacial Surgery 56 (2018) 810-13.

30. Wang S, XiaoJ, Liu L, Lin Y, Li X, Tang W et al. Orbital floor reconstruction: a retrospective study of 21 cases. OralSurg Oral Med Oral Pathol Oral Radiol Endod 2008;106:324-30. 